\title{
Tetrandrine Suppresses Transient Receptor Potential Cation Channel Protein 6 Overexpression- Induced Podocyte Damage via Blockage of RhoA/ROCKI Signaling
}

This article was published in the following Dove Press journal:

Drug Design, Development and Therapy

Jin $\mathrm{Yu}$

Caifeng Zhu

Jiazhen Yin

Dongrong Yu

Feng Wan

Xuanli Tang

Xue Jiang

Department of Nephrology, Guangxing Hospital Affiliated to Zhejiang Chinese Medical University (Key Laboratory of

Zhejiang Province, Management of Kidney Disease), Hangzhou 310007, People's Republic of China
Correspondence: Xue Jiang

Department of Nephrology, Guangxing Hospital Affiliated to Zhejiang Chinese

Medical University (Key Laboratory of

Zhejiang Province, Management of Kidney

Disease), Hangzhou 310007, People's

Republic of China

Email monica_jiang@I63.com
Objective: Podocyte damage is common in many renal diseases characterized by proteinuria. Transient receptor potential cation channel protein 6 (TRPC6) plays an important role in renal function through its regulation of intracellular $\mathrm{Ca}^{2+}$ influx and RhoA/ROCK pathways. Chinese herb Stephania tetrandra, with the main active component being tetrandrine, has been used for the treatment of various kidney diseases for several years and has shown a positive effect. This study aimed at investigating the effect and mechanism of tetrandrine in podocyte damage induced by high expression of TRPC6.

Methods: Immortalized, differentiated murine podocytes, MPC5 were treated with valsartan $(0-800 \mu \mathrm{M})$ and tetrandrine $(0-40 \mu \mathrm{M})$ for $48 \mathrm{~h}$. The maximum safe concentrations of valsartan and tetrandrine were selected using a cell viability assay. MPC5 podocytes stably expressing TRPC6 were constructed using a lentivirus packaging system, followed by treatment with valsartan, tetrandrine, and Y-27632 for $48 \mathrm{~h}$ and U73122 $(10 \mu \mathrm{M})$ for $10 \mathrm{~min}$. The RhoA/ ROCK pathway and podocyte-specific proteins (nephrin and synaptopodin) levels were quantified. Podocyte apoptosis and intracellular $\mathrm{Ca}^{2+}$ concentration were measured.

Results: Maximum safe concentrations of $100 \mu \mathrm{M}$ valsartan and $10 \mu \mathrm{M}$ tetrandrine showed no observable toxicity in podocytes. MPC5 podocytes stably expressing TRPC6 had higher intracellular $\mathrm{Ca}^{2+}$ influx, apoptotic percentages, and expression of RhoA/ROCK proteins, but lower expression of nephrin and synaptopodin proteins. U73122 treatment for $10 \mathrm{~min}$ did not inhibit TRPC6, but suppressed RhoA/ROCK protein. Y-27632 decreased ROCK1 expression, but did not influence the expression of TRPC6 protein. Both $100 \mu \mathrm{M}$ valsartan and $10 \mu \mathrm{M}$ tetrandrine for $48 \mathrm{~h}$ significantly inhibited intracellular $\mathrm{Ca}^{2+}$ influx, apoptosis, and RhoA/ROCK pathway, and increased nephrin and synaptopodin proteins in podocytes stably expressing TRPC6.

Conclusion: Elevated TRPC6 expression can lead to podocyte injury by inducing intracellular $\mathrm{Ca}^{2+}$ influx and apoptosis of podocytes, and this effect may be mediated by activation of the RhoA/ ROCK1 pathway. Tetrandrine can alleviate podocyte injury induced by TRPC6 expression through inhibition of the RhoA/ROCK pathway, suggesting a protective role in podocyte damage.

Keywords: tetrandrine, transient receptor potential cation channel protein 6 , podocyte, RhoA/ROCK pathway

\section{Introduction}

Numerous studies have shown that podocyte damage plays a critical role in the pathogenesis of various kidney diseases including diabetic nephropathy, focal segmental glomerulosclerosis (FSGS) and $\operatorname{IgA}$ nephropathy (IgAN). ${ }^{1-3}$ Podocyte 
damage triggers a series of changes like increases in mesangial extracellular matrix, kidney injury, glomerulosclerosis and renal failure. ${ }^{4,5}$ Therefore, prevention of podocyte damage is crucial for the treatment of kidney diseases.

Transient receptor potential cation channel protein 6 (TRPC6) is colocalized in the kidney podocyte glomerular slit diaphragm with the protein, nephrin. ${ }^{3,6}$ TRPC6 plays a role in renal functions and diseases by mediating calcium influx via its interaction with calmodulin. ${ }^{3,6-8}$ TRPC6 channel is induced by phospholipase C (PLC) stimulation and its overexpression in podocytes induces intracellular $\mathrm{Ca}^{2+}$ influx, which then activates RhoA, induces cytoskeleton rearrangement and the apoptosis of podocytes. $6,8,9$ TRPC6 gain-of-function mutations increase intracellular $\mathrm{Ca}^{2+}$ load, and podocyte cell death. It can also lead to hereditary FSGS and is implicated in acquired glomerular proteinuric diseases. ${ }^{10,11}$ The downregulation or inhibition of TRPC6 or RhoA pathway reverses all these deficits, ${ }^{9,12}$ suggesting that targeting of TRPC6 could be protective in podocyte damage.

Tetrandrine, a bisbenzylisoquinoline alkaloid, the main active component isolated from the Chinese herbal radix Stephania tetrandra has a definite anti-hypertensive, antiinflammatory, and anti-cognitive impairment effect. ${ }^{13,14}$ Tetrandrine, one of the most active components in Fangji Huangqi Tang (FHT), a well-known traditional Chinese medicine prescription for treatment of nephrotic syndrome, exhibits anti-inflammatory effects against edema and swelling in rat paws. ${ }^{15-17}$ In addition, our previous clinical trials have shown that using a traditional Chinese medicinal herb recipe including $S$. Tetrandra significantly alleviated proteinuria and improved the renal function in patients with IgAN. ${ }^{18,19}$ It has been shown in that effects of tetrandrine are related to the blocking of calcium channels in various cell types, including cardiomyocytes, ventricular cells, vascular smooth muscle cells (VSMCs), cancer cells, and hepatocytes. ${ }^{20-23}$ Tetrandrine exhibits its protective effects on various disorders via pathways including RhoA, mitogen-activated protein kinase (MAPK), and Wnt/ $\beta$-catenin. ${ }^{24,25}$ Previous studies have shown that the inhibition of $\mathrm{Ca}^{2+} /$ calmodulin-dependent protein kinase II $\delta$ and calmodulin-dependent phosphodiesterase activity were related to the effect of tetrandrine. $^{26,27}$ However, the blocking effect of tetrandrine on podocytes has not been reported until now.

According to the above evidence, we speculated that TRPC6 overexpression induces intracellular $\mathrm{Ca}^{2+}$ influx and apoptosis in podocytes by activating RhoA/Rho kinase (ROCK) signaling. Tetrandrine exhibit protective effects on podocytes damage via blockade of TRPC6-mediated intracellular $\mathrm{Ca}^{2+}$ influx and RhoA/Rock signaling. To test this hypothesis, we performed in vitro experiments using TRPC6-overexpressing podocytes. A widely used nonpeptide angiotensin receptor antagonist valsartan, which has crucial roles in protecting podocytes, was used as a positive control. ${ }^{28,29}$ U73122 (U73122 inhibits phospholipase $\mathrm{C}$, so that less diacylglycerol is produced and the opening of TRPC6 channels is reduced) and Y-27632 (inhibitor of ROCK, a downstream effector of RhoA) were also used as positive controls. Podocytes differentiation, cellular $\mathrm{Ca}^{2+}$ release, apoptosis and RhoA/ROCK signaling were investigated. Our study would provide novel insights in the protective effect of tetrandrine in podocyte injury.

\section{Materials and Methods MPC5 Cell Culture}

Immortalized differentiated murine podocytes (MPC5) were obtained from Dr. Peter Mundel (Mount Sinai School of Medicine, New York, USA) and maintained in RPMI-1640 (Gibco BRL, Grand Island, NJ, USA) supplemented with 10\% HyClone fetal bovine serum (FBS, Gibco BRL), and $100 \mathrm{U} / \mathrm{mL}$ interferon (IFN)- $\gamma$ (PeproTech, Inc., Rocky Hill, NJ, USA) at $33^{\circ} \mathrm{C}$ with $5 \%$ $\mathrm{CO}_{2}$ for proliferation. Cell differentiation was induced by incubating in RPMI-1640 without IFN- $\gamma$ at $37^{\circ} \mathrm{C}$ with $5 \%$ $\mathrm{CO}_{2}$ for 14 days. For the identification of MPC5 differentiation, nephrin immunofluorescence assay was performed.

\section{TRPC6 Expression Plasmid Construction, Transfection and Screening of TRPC6 Stable Cell Line}

The TRPC6-overexpressing plasmid was constructed by cloning the full length of the CDS of TRPC6 gene (Kingsley biological co, LTD) into EcoRI/BamHI sites of pCDH-GFP-PURO vector (System Biosciences, Palo Alto, CA, USA). The plasmids were packaged into lentivirus (Shanghai GeneChem. CO., Ltd, Shanghai, China) in HEK 293T packaging cells (Shanghai GeneChem. CO., Ltd). In brief, MPC5 podocytes were seeded in 6-well plates $\left(1.0 \times 10^{5}\right)$ and incubated with polybrene $(6 \mu \mathrm{g} / \mathrm{mL}$; SigmaAldrich, St. Louis, MO, USA) in combined with lentivirus containing TRPC6-overexpressing plasmid (multiplicity of 
infection of 10 to 30 ) or blank lentivirus vector as a negative control (NC). Positive cells were selected for in RPMI-1640 containing $1 \mu \mathrm{g} / \mathrm{mL}$ puromycin until the purity of TRPC6-overexpressing cells reached $80 \%$. The TRPC6 stable cell line was confirmed using TRPC6 protein and mRNA expression, and was used for further experiments. MPC5 podocytes were divided into the TRPC6 group (expressing lentivirus containing TRPC6overexpressing plasmid) or the NC group (containing blank lentivirus vector).

\section{Cell Treatments}

TRPC6-overexpressing cells were treated with a series of valsartan (0-800 $\mu \mathrm{M}$; Meilun Biotech Co., Ltd., Dalian, China) and tetrandrine (0-40 $\mu \mathrm{M}$; Meilun) concentrations at $37^{\circ} \mathrm{C}$ for $48 \mathrm{~h}$. For U73122 (inhibitor of PLC, Calbiochem, La Jolla, CA, USA) treatment, cells were incubated with $\mathrm{U} 73122(10 \mu \mathrm{M})$ for $10 \mathrm{~min}^{30,31}$ or Y-27632 (10 $\mathrm{mmol} / \mathrm{L})$ for $30 \mathrm{~min}$ followed by harvesting or continued incubation in fresh medium for $48 \mathrm{~h}$. Each experiment was performed in triplicate.

\section{Cell Proliferation Analysis}

For the cell viability assay, the RPMI-1640 medium was replaced by FBS-free medium containing 10\% CCK-8 working solution $(5 \mathrm{mg} / \mathrm{mL}$; Beyotime Institute of Biotechnology, Shanghai, China) and incubated in the dark for $1 \mathrm{~h}$. A microplate reader (BioTek Instruments, Winooski, VT, USA) was used for recording the absorbance (OD450 $\mathrm{nm}$ ).

\section{In vitro Cytotoxicity Assay}

MPC5 podocytes were incubated for $48 \mathrm{~h}$. The quantitation of lactate dehydrogenase (LDH) release in culture was detected using Cytotoxicity LDH Assay Kit-WST (Dojindo Laboratories, Kumamoto Japan) according to the manufacturer's instructions.

\section{Cell Apoptosis}

MPC5 podocytes $\left(5 \times 10^{5}\right.$ cells $\left./ \mathrm{mL}\right)$ were seeded in 6 -well plates followed by incubation with valsartan and tetrandrine for $48 \mathrm{~h}$ or with $\mathrm{U} 73122$ for $10 \mathrm{~min}$. Apoptosis analysis was performed using Annexin V/PI fluorescent double staining (BD Biosciences, San Jose, CA, USA) according to manufacturers' instructions. A BD FACS Calibur ${ }^{\mathrm{TM}}$ flow cytometry (BD Biosciences) was used for the analysis. Each experiment was performed in triplicate.

\section{Confocal Calcium Imaging}

MPC5 podocytes were seeded in 24-well plates and treated with valsartan and tetrandrine for $48 \mathrm{~h}$ or with U73122 for $10 \mathrm{~min}$, then incubated with RPMI-1640 supplemented with $10 \mu \mathrm{M}$ Fluo-3AM (Dojindo Laboratories) for $30 \mathrm{~min}$ in the dark. Cells were then washed with PBS and maintained in medium and immediately excited (488 nm) using a Zeiss laser confocal imaging system (Zeiss, Thornwood, NY, USA).

\section{Reverse Transcription Quantitative PCR (RT-qPCR)}

After incubating with valsartan or tetrandrine for $48 \mathrm{~h}$ or with U73122 for $10 \mathrm{~min}$, cells $\left(5-10 \times 10^{6}\right)$ were harvested and total RNA were extracted using TRIzol according to the manufacturers' instruction (Qiagen). cDNA templates were achieved by reverse transcribing the RNA using $5 \mathrm{x}$ primeScript RT Master MIXperfect Real Time; TAKARA, Tokyo, Japan). RT-qPCR reaction was performed using Power SYBR Green PCR Master Mix (Thermo Fisher Scientific, Passau, Germany) using synthesized primers (Table 1) and following reaction conditions: $50^{\circ} \mathrm{C}$ for $3 \mathrm{~min} ; 95^{\circ} \mathrm{C}$ for $3 \mathrm{~min}$; 40 cycles of $95^{\circ} \mathrm{C}$ for $10 \mathrm{~s}, 60^{\circ} \mathrm{C}$ for $30 \mathrm{~s}$. The $2^{-\triangle \Delta \mathrm{ct}}$ methods was used to calculate the relative expression levels of targeted genes. GAPDH was selected as the reference internal gene.

\section{Western Blotting Assay}

Total protein samples were extracted from cells after incubating with valsartan, tetrandrine and Y-27632 for $48 \mathrm{~h}$ or with U73122 for $10 \mathrm{~min}$. RIPA lysis buffer (Beyotime) with $1 \mathrm{mM}$ phenylmethanesulfonyl fluoride (PMSF; Beyotime) was used for cell lysis. The supernatant was transferred to a centrifuge tube for vortexing. Protein lysates were centrifuged at $4{ }^{\circ} \mathrm{C}$, $12,000 \times \mathrm{g}$ for $10 \mathrm{~min}$. Protein samples were subjected to

Table I The Sequences of Primers Used in This Study

\begin{tabular}{|l|l|l|}
\hline Gene Name & Primers & Sequences \\
\hline GAPDH & $\begin{array}{l}\text { Forward } \\
\text { Reverse }\end{array}$ & $\begin{array}{l}\text { GGTGAAGGTCGGTGTGAACG } \\
\text { CTCGCTCCTGGAAGATGGTG }\end{array}$ \\
\hline TRPC6 & $\begin{array}{l}\text { Forward } \\
\text { Reverse }\end{array}$ & $\begin{array}{l}\text { AGCCAGGACTATTTGCTGATGG } \\
\text { AACCTTCTTCCCTTCTCACGA }\end{array}$ \\
\hline RhoA & $\begin{array}{l}\text { Forward } \\
\text { Reverse }\end{array}$ & $\begin{array}{l}\text { CAGAGGTCTATGTGCCCAC } \\
\text { ATAAACCAACTCTACCTGCT }\end{array}$ \\
\hline ROCKI & $\begin{array}{l}\text { Forward } \\
\text { Reverse }\end{array}$ & $\begin{array}{l}\text { GACTGGGGACAGTTTTGAGAC } \\
\text { GGGCATCCAATCCATCCAGC }\end{array}$ \\
\hline
\end{tabular}


$10 \%$ SDS-PAGE for separation and then transferred onto polyvinylidene difluoride (PVDF) membranes according to standards methods. PVDF membranes were blocked with skim milk powder, and incubated with primary antibodies. against TRPC6 (1: 1000, Santa Cruz), Synaptopodin (1: 100; Santa Cruz), Nephrin (1: 100; Proteintech), ROCK1 (1: 1000; Proteintech), anti-RhoA antibody (1: 1000; Proteintech, Wuhan, China), and $\beta$-actin (1: 1000; Santa Cruz) at $4{ }^{\circ} \mathrm{C}$ overnight. Activated RhoA was determined by measuring membrane-bound RhoA (GTP-RhoA) using the GST rhotekin-Rho binding domain that specifically pulls down activated RhoA, Secondary antibodies were peroxidase AffiniPure donkey anti-mouse or goat anti-rabbit $\operatorname{IgG}(\mathrm{H}+\mathrm{L}$; Jackson ImmunoResearch Laboratories, West Grove, PA, USA). The blots were developed using ECL system (Sharebio, Shanghai, China) and analyzed using the TanonImage software (Tanon, Shanghai, China).

\section{Statistical Analysis}

All the data was represented by the mean \pm standard deviation. Statistical analysis for the differences among groups was performed using a one-way ANOVA in GraphPad Prism 6 (GraphPad Software, San Diego, CA). Differences between two groups were analyzed using a $t$-test. For all analysis, $\mathrm{p}<0.05$ was considered statistically significant.

\section{Results}

\section{Cytotoxicity of Valsartan, Tetrandrine,} Y-27632 and U73 122, and Selection of Optimal Valsartan and Tetrandrine

\section{Concentrations}

As indicated by the quantitation of $\mathrm{LDH}$ release, the administration of $100 \mu \mathrm{M}$ valsartan and $10 \mu \mathrm{M}$ tetrandrine for $48 \mathrm{~h}$ showed no toxicity in MPC5 podocytes, while Y-27632 (10 mmol/L) for $30 \mathrm{~min}$ and $10 \mu \mathrm{M} \mathrm{U} 73122$ for $10 \mathrm{~min}$ followed with fresh medium for $48 \mathrm{~h}$ showed obvious toxicity (Figure 1B). We used CCK to detect cell viability to confirm these findings (Figure 1A). For the following experiments valsartan and tetrandrine were administered at $100 \mu \mathrm{M}$ and $10 \mu \mathrm{M}$, respectively. These data suggest that valsartan and tetrandrine do not exhibit MPC5 podocyte toxicity at the concentrations tested.

\section{Tetrandrine Prevents TRPC6-Induced Intracellular $\mathrm{Ca}^{2+}$ Influx in Podocytes}

Using plasmid transfection, we constructed a podocyte injury model through TRPC6-overexpression. In the TRPC6 group, we found that the expression of TRPC6 significantly increased at the mRNA and protein levels compared to that in the $\mathrm{NC}$ (containing blank lentivirus vector) and blank (normal MPC5 podocyte) groups $(\mathrm{p}<0.001$; Figure $2 \mathrm{~A}$ and B). Use of fluo-3AM detected $\mathrm{Ca}^{2+}$ influx in podocytes suggested that overexpression of TRPC6 increased intracellular $\mathrm{Ca}^{2+}$ influx, while the administration of tetrandrine attenuated it. There were no obvious increases of $\mathrm{Ca}^{2+}$ influx in the blank and $\mathrm{NC}$ control groups (Figure 3). Compared with U73122, valsartan and tetrandrine had stronger inhibitory effects on TRPC6-induced intracellular $\mathrm{Ca}^{2+}$ influx $(\mathrm{p}<0.05)$. These data suggest that tetrandrine is highly efficient in suppressing TRPC6induced intracellular $\mathrm{Ca}^{2+}$ influx in podocytes.

\section{Tetrandrine Prevents Apoptosis in TRPC6-Overexpressing Podocytes}

Flow cytometry analysis showed that the exogenous overexpression of TRPC6 significantly increased the apoptotic percentage of podocytes compared with the blank and NC
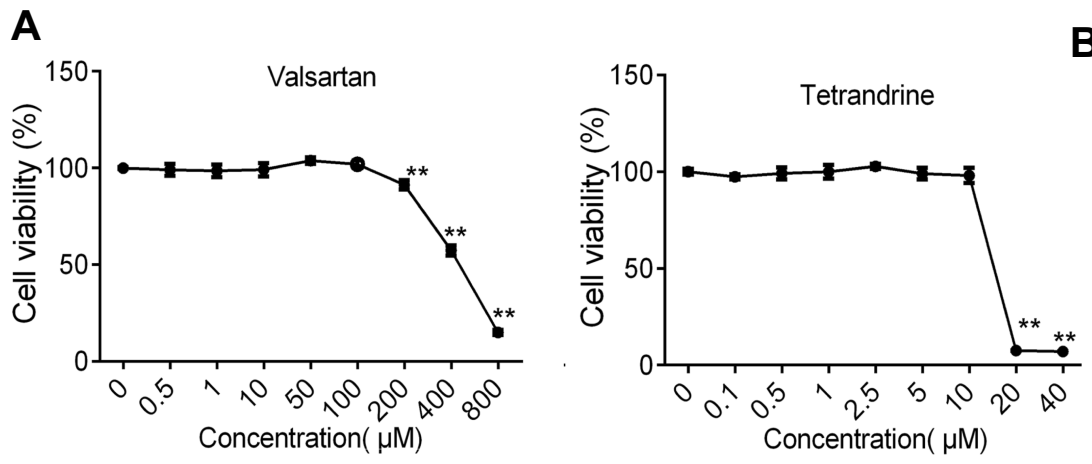

B

Figure I Optimization of drug concentrations. (A) Cell viability of MPC5 podocytes under treatment with different concentrations of drugs detected by CCK8. (B) The LDH cytotoxicity assay in MPC5 podocytes treated with different drugs. **P<0.0I NC: containing blank lentivirus vector. blank: normal MPC5 podocyte.TRPC6 group: TRPC6-overexpressing. 

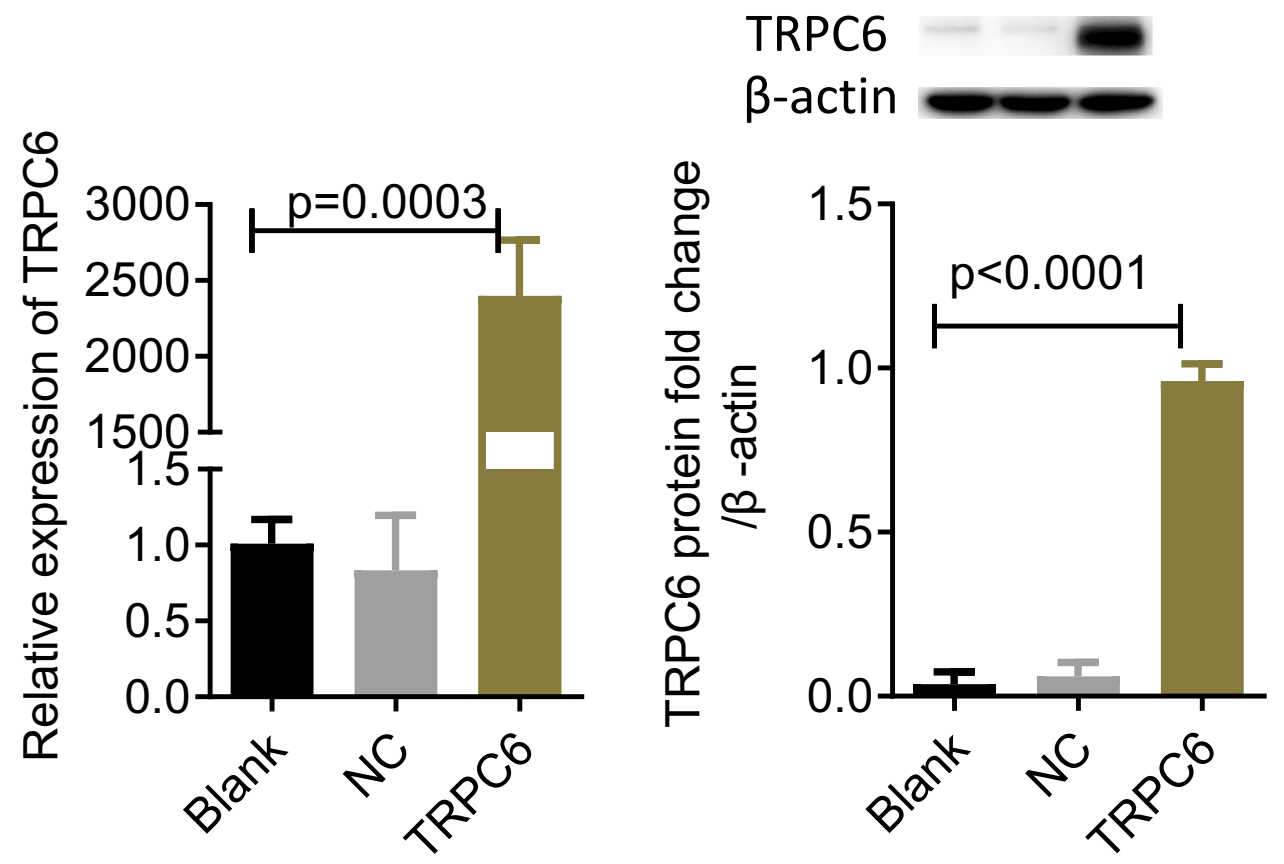

Figure 2 Detection of TRPC6 mRNA and protein expression in podocyte transfection of lentivirus packaging plasmid, respectively. (A) detection of TRPC6 mRNA expression by RT-qPCR. (B) detection of TRPC6 protein levels by western-blot. NC: containing blank lentivirus vector. blank: normal MPC5 podocyte.TRPC6 group: TRPC6-overexpressing.

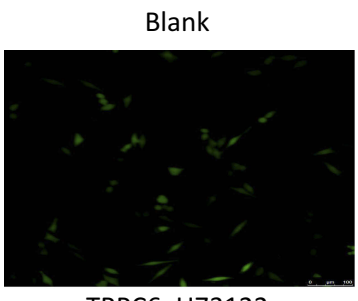

TRPC6+U73122

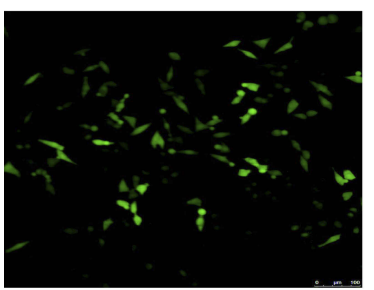

NC

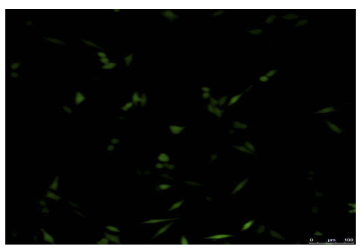

TRPC6+Valsartan

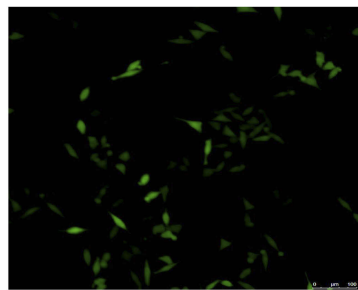

TRPC6

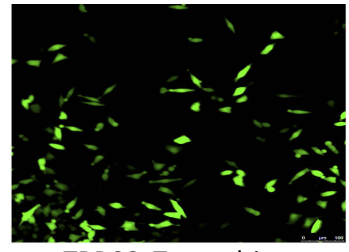

TRPC6+Tetrandrine

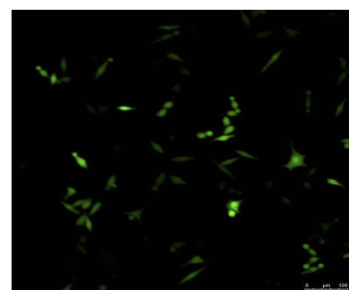

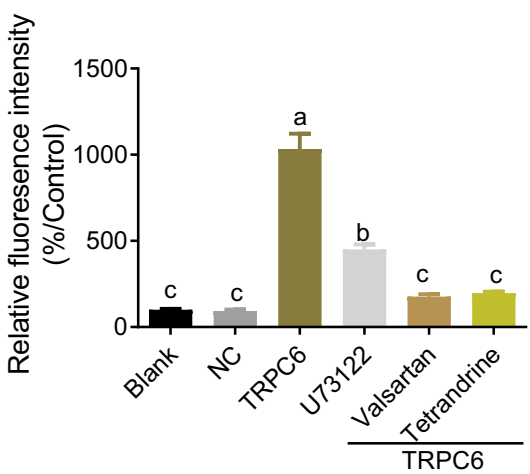

Figure 3 Tetrandrine inhibits TRPC6 overexpression-induced intracellular $\mathrm{Ca}^{2+}$ influx in MPC5 podocytes. Fluorescence image of fluo-3AM loaded cells (green) indicates intracellular $\mathrm{Ca}^{2+}$ influx. Differences were analyzed using one-way ANOVA. Significant differences with $\mathrm{p}<0.05$ are indicated by different letters. NC: containing blank lentivirus vector. blank:normal MPC5 podocyte. TRPC6 group: TRPC6-overexpressing.

groups $(19.33 \pm 0.67 \%, \quad 0.83 \pm 0.17 \%, \quad 1.33 \pm 0.33, \mathrm{p}<0.05$,

Figure 4), suggesting that TRPC6 expression is required for MPC5 apoptosis. In contrast, the TRPC6-induced apoptosis was decreased by ally drugs, especially by tetrandrine. The apoptotic percentage of MPC5 podocytes in TRPC6+tetrandrine-treated cells $(10.00 \pm 0.58 \%)$ was significantly lower than that of TRPC6+U73122 (14.23 $\pm 0.74 \%, \quad \mathrm{p}<0.05)$ and TRPC6+valsartan-treated cells $(14.00 \pm 0.32 \%, \mathrm{p}<0.05$, Figure 4). These data suggest that tetrandrine suppresses TRPC6-induced apoptosis in podocytes.

\section{Tetrandrine Prevents TRPC6-Induced} Podocyte Injury via Activating RhoA/ ROCKI Signaling

We performed further drug intervention and confirmed that valsartan and U73122 did not influence TRPC6 mRNA 

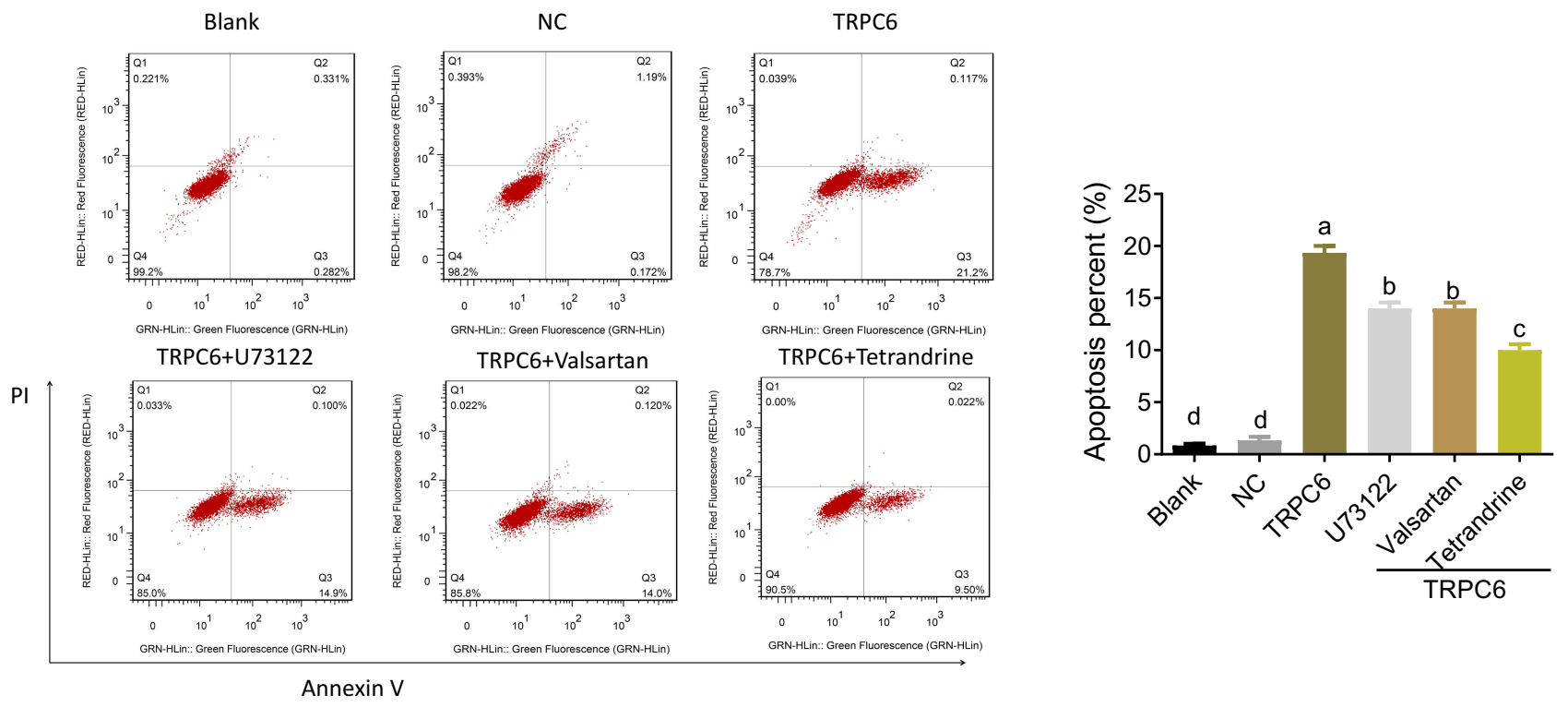

Figure 4 Tetrandrine prevents TRPC6 overexpression-induced apoptosis in MPC5 podocytes. Apoptosis was quantified using flow cytometry. Differences were analyzed using one-way ANOVA. Different letters show significant differences with $\mathrm{p}<0.05$. NC: containing blank lentivirus vector. blank:normal MPC5 podocyte.TRPC6: TRPC6overexpressing.

expression, while the expression of TRPC6 was decreased after treatment with tetrandrine (Figure 5A). RhoA and ROCK1 mRNA expression were increased in the TRPC6 group. Furthermore, the expression of RhoA and ROCK1 were significantly reduced in tetrandrine-treated cells, compared to cells treated with U73122 or valsartan $(\mathrm{p}<0.05)$.

Western blot analysis confirmed the striking decrease in TRPC6, RhoA-GTP/RhoA and ROCK1 protein levels by tetrandrine (Figure 5B). Similar to valsartan, U73122 decreased RhoA-GTP/RhoA and ROCK1 expression, but did not influence the expression of TRPC6 protein. We also detected that the overexpression of TRPC6 resulted in downregulation of nephrin and synaptopodin; however, this is reversed upon administration of tetrandrine, U73122 and valsartan. Together, these data suggest that TRPC6 overexpression promotes podocytes injury via RhoA/ROCK1 signaling, which is reversed by the administration of tetrandrine. Moreover, Western blot analysis confirmed that the ROCK inhibitor Y-27632 decreased ROCK1 signaling, but did not affect TRPC6 expression. Treatment with Y-27632 partially rescued the decrease in synaptopodin induced by TRPC6 overexpression, but the effect was not as robust as that of tetrandrine (Figure 6).

\section{Discussion}

Tetrandrine exhibits its protective effect on various disorders via pathways including inactivation of RhoA/ROCK signaling. ${ }^{24}$ Our present study highlighted the protective effects of tetrandrine on TRPC6-induced podocyte injury via inhibition of intracellular $\mathrm{Ca}^{2+}$ influx, podocyte apoptosis, and RhoA/ROCK pathway. In addition, it showed equivalent or higher efficiency than valsartan in protecting against TRPC6-induced podocyte damage.

Many studies have shown that Angiotensin II (Ang II) can induce reorganization of the actin cytoskeleton in podocytes via activation of the RhoA/ROCK signaling pathway. ${ }^{32}$ Valsartan is widely used as an angiotensin receptor antagonist. The protective effect of valsartan on podocyte damage has been widely reported. ${ }^{33-36}$ At the same time, ACEI/ARB functions to decrease urinary protein. Independently of hypotension, valsartan increased the expression of nephrin and podocin in renal tissues ${ }^{36}$ and promoted the repairs of podocyte membranes via an increase in P-Cadherin and Wilms tumor-1 (WT-1) and by decreasing inflammatory factors including interleukin (IL)-1 $\beta$, tumor necrosis factor (TNF)- $\alpha$, and IL- $6 .{ }^{35}$ Studies have also confirmed that losartan (ARB) can reduce proteinuria and protect renal function by reducing TRPC6 expression in a rat model of adriamycin nephropathy. ${ }^{37}$ Our present study confirmed that valsartan plays protective roles in TRPC6 overexpression-mediated podocyte damage via blockage of intracellular $\mathrm{Ca}^{2+}$ influx, thereby inhibiting the RhoA/ROCK signaling pathway and ultimately podocyte apoptosis. Furthermore, we showed that valsatran rescued the TRPC6 overexpression-induced 

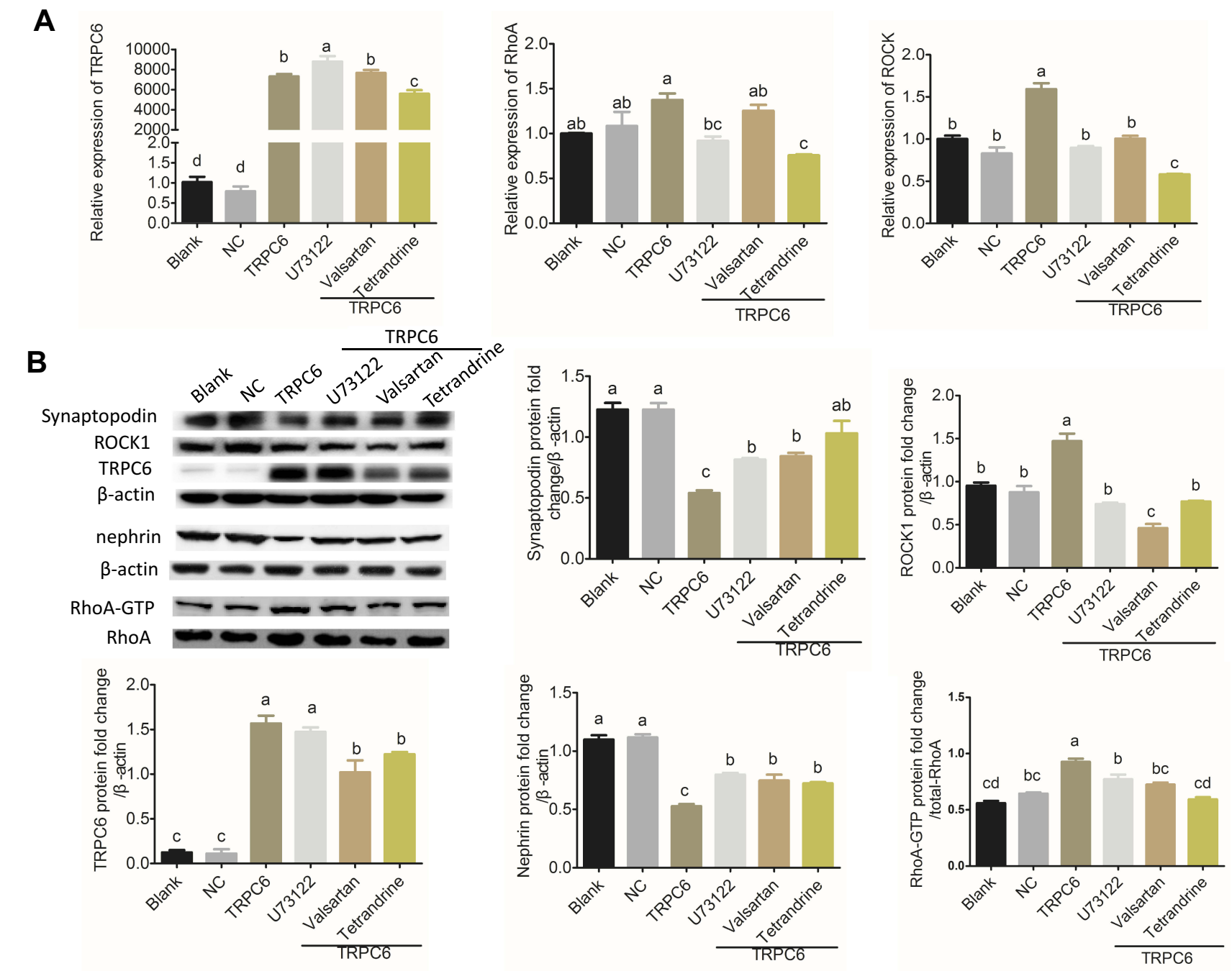

Figure 5 TRPC6 activates RhoA/ROCKI signaling. (A) Analysis of of TRPC6, RhoA and ROCKI mRNA by RT-qPCR analysis. (B) Analysis of TRPC6, RhoA-GTP/RhoA and ROCKI, nephrin and synaptopodin protein levels by Western blot. Differences were analyzed using one-way ANOVA. Significant differences with $p<0.05$ are indicated by different letters. NC: containing blank lentivirus vector. blank:normal MPC5 podocyte.TRPC6: TRPC6-overexpressing.

downregulation of critical renal proteins, nephrin and synaptopodin.

In comparison with valsartan, tetrandrine treatment exhibited superior effects on alleviating TRPC6-mediated damage and apoptosis in podocytes, with lower concentrations. Tetrandrine inhibited TRPC6 overexpression-induced podocyte apoptosis and intracellular $\mathrm{Ca}^{2+}$ influx. It has been shown that the protective effects in various cell types are related to calcium channel inhibition via calmodulindependent phosphodiesterase activity and a series of pathways including inactivation of the RhoA pathway and activation of PI3K/Akt pathway. ${ }^{22-24,27}$ Our present study demonstrated that the activation of the RhoA/ROCK pathway caused by TRPC6-mediated intracellular $\mathrm{Ca}^{2+}$ influx was crucial for podocyte apoptosis. In contrast, TRPC6 protein associated with the cytoskeleton to modulate cytoskeletal organization via the ion-transport-dependent pathway, ${ }^{38}$ while activation of the RhoA pathway can also induce the rearrangement of podocyte-associated actin. These data suggest that tetrandrine selectively inhibits the overexpression of TRPC6 and interferes with the downstream pathways to restore the downregulated nephrin and synaptopodin. Additionally, the protective effect of tetrandrine was more robust than that of valsartan.

ROCK inhibitors (Y-27632) can inhibit the activation of ROCK1 but cannot inhibit the overexpression of TRPC6 and the $\mathrm{Ca}^{2+}$ flow mediated by it. Y-27632 improved the decrease in synaptopodin induced by TRPC6 overexpression, but the effect was weaker than that of tetrandrine. Increased expression of TRCP6 results in $\mathrm{Ca}^{2+}$ influx, but 

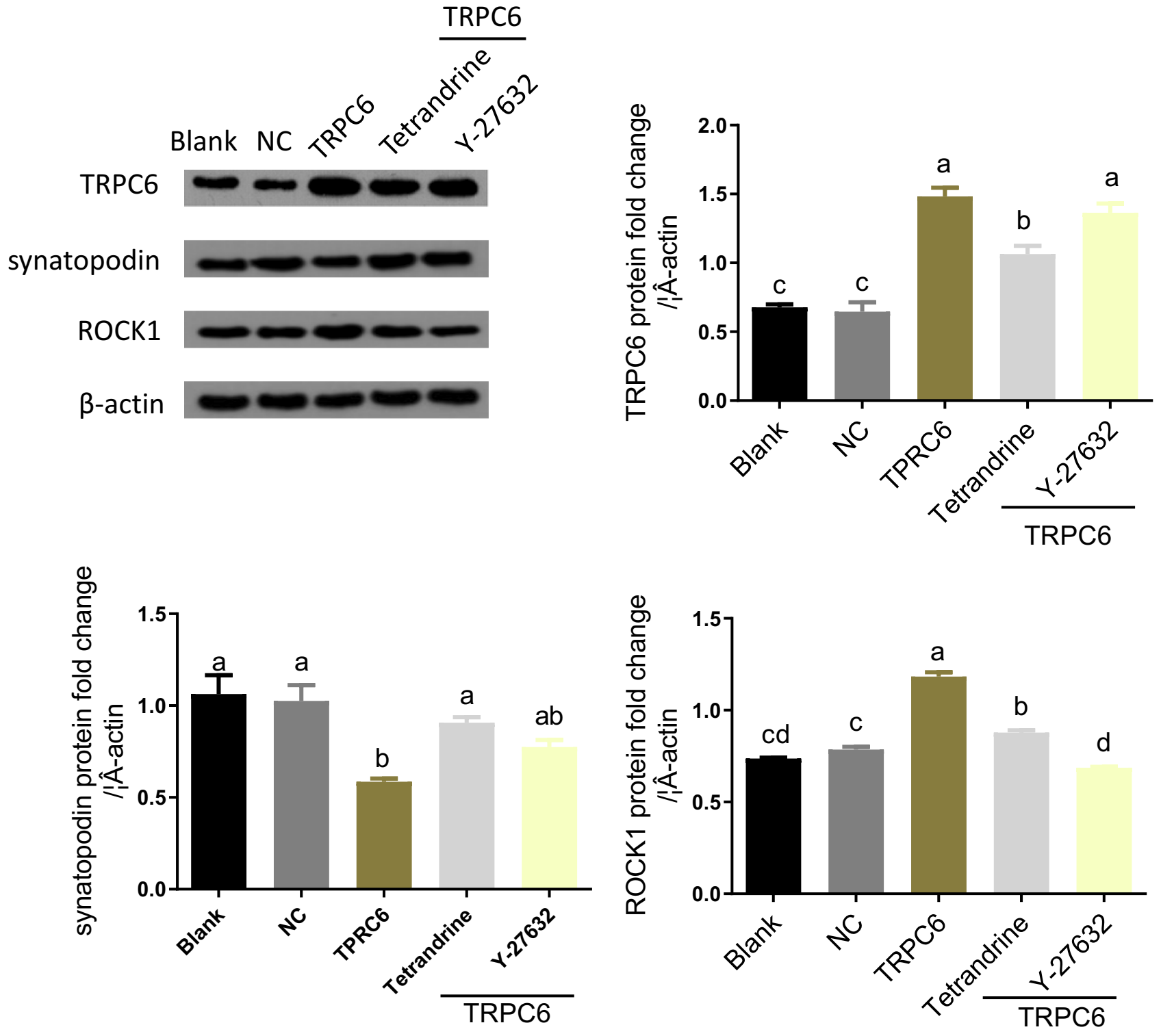

Figure 6 Analysis of TRPC6, ROCK I, and synaptopodin protein levels by Western blot in different groups when cells were treated with Y-27632. Differences were analyzed using one-way ANOVA. Significant differences with $\mathrm{p}<0.05$ are indicated by different letters. NC: containing blank lentivirus vector. blank: normal MPC5 podocyte. TRPC6: TRPC6-overexpressing.

the downstream signaling pathways triggered by TRCP6 are complex and have not been fully studied, therefore there may be other approaches besides RhoA/ROCK, since ROCK is not the only effector of RhoA. Thus, this study speculated that the role of tetrandrine is more focused on inhibiting TRPC6 overexpression upstream of the pathway and reducing the influx of $\mathrm{Ca}^{2+}$. Besides blocking of RhoA/ ROCK1 signaling, the mechanism of protection against TRPC6-mediated podocyte injury by tetrandrine requires further investigation. We also used U73122 in parallel to inhibit TRPC6 channel opening and observed that U73122 administration did not inhibit the overexpression of TRPC6 in podocytes, while it did have an effect on reducing intracellular $\mathrm{Ca}^{2+}$ influx and inhibiting RhoA/ROCK activation, the long-term administration showed increased podocyte toxicity.

\section{Conclusion}

In conclusion, our study confirmed the protective effect of tetrandrine in podocytes damage and showed more positive effects than the previously elucidated valsartan. Tetrandrine inhibited TRPC6-overexpression-induced intracellular $\mathrm{Ca}^{2+}$ influx, podocyte apoptosis and enhanced podocyte growth via inhibition of the RhoA/ROCK pathway. In sum, these 
results suggest that tetrandrine plays an important role in protecting podocyte injury.

\section{Data Sharing Statement}

All data generated or analyzed during this study are included in this published article.

\section{Funding}

This study is supported by Hangzhou Agricultural and Social Development Research Project (2019120B136 and 20170533B86).

\section{Disclosure}

The authors declare that they have no competing interests.

\section{References}

1. Le W, Liang S, Hu Y, et al. Long-term renal survival and related risk factors in patients with IgA nephropathy: results from a cohort of 1155 cases in a Chinese adult population. Nephrol Dial Transplant. 2011;27:1479-1485. doi:10.1093/ndt/gfr527

2. Khalilpourfarshbafi M, Hajiaghaalipour F, Selvarajan KK, Adam A. Mesenchymal stem cell-based therapies against podocyte damage in diabetic nephropathy. J Tissue Eng Regen Med. 2017;14:201-210. doi:10.1007/s13770-017-0026-5

3. Ilatovskaya DV, Blass G, Palygin O, et al. A NOX4/TRPC6 pathway in podocyte calcium regulation and renal damage in diabetic kidney disease. J Am Soc Nephrol. 2018;29:1917-1927. doi:10.1681/ASN.20 18030280

4. Lopes TG, de Souza ML, da Silva VD, et al. Markers of renal fibrosis: how do they correlate with podocyte damage in glomerular diseases? PLoS One. 2019;14:e0217585. doi:10.1371/journal.pone. 0217585

5. Chen Y, Lin L, Tao X, Song Y, Cui J, Wan J. The role of podocyte damage in the etiology of ischemia-reperfusion acute kidney injury and post-injury fibrosis. BMC Nephrol. 2019;20:106. doi:10.1186/ s12882-019-1298-x

6. Riehle M, Büscher AK, Gohlke B-O, et al. TRPC6 G757D loss-offunction mutation associates with FSGS. $J$ Am Soc Nephrol. 2016;27:2771-2783. doi:10.1681/ASN.2015030318

7. Szabo T, Ambrus L, Zakany N, Balla G, Biro T. Regulation of TRPC6 ion channels in podocytes-Implications for focal segmental glomerulosclerosis and acquired forms of proteinuric diseases. Acta Physiol Hung. 2015;102:241-251. doi:10.1556/036.102.2015.3.2

8. Reiser J, Polu KR, Möller CC, et al. TRPC6 is a glomerular slit diaphragm-associated channel required for normal renal function. Nat Genet. 2005;37:739. doi:10.1038/ng1592

9. Ilatovskaya DV, Staruschenko A. TRPC6 channel as an emerging determinant of the podocyte injury susceptibility in kidney diseases. Am J Physiol Renal Physiol. 2015;309:F393-F397. doi:10.1152/ ajprenal.00186.2015

10. Jiang L, Ding J, Tsai $\mathrm{H}$, et al. Over-expressing transient receptor potential cation channel 6 in podocytes induces cytoskeleton rearrangement through increases of intracellular $\mathrm{Ca} 2+$ and RhoA activation. Exp Biol Med. 2011;236:184-193. doi:10.1258/ebm.2010.010237

11. Yang H, Zhao B, Liao C, et al. High glucose-induced apoptosis in cultured podocytes involves TRPC6-dependent calcium entry via the RhoA/ROCK pathway. Biochem Biophys Res Commun. 2013;434: 394-400. doi:10.1016/j.bbrc.2013.03.087
12. Yao XM, Liu YJ, Wang YM, et al. Astragaloside IV prevents high glucose-induced podocyte apoptosis via downregulation of TRPC6. Mol Med Rep. 2016;13:5149-5156. doi:10.3892/mmr.2016.5167

13. Huang Y-L, Cui S-Y, Cui X-Y, et al. Tetrandrine, an alkaloid from S. tetrandra exhibits anti-hypertensive and sleep-enhancing effects in SHR via different mechanisms. Phytomedicine. 2016;23:1821-1829.

14. Ma H, Yao L, Pang L, Li X, Yao Q. Tetrandrine ameliorates sevoflurane-induced cognitive impairment via the suppression of inflammation and apoptosis in aged rats. Mol Med Rep. 2016;13: 4814-4820. doi:10.3892/mmr.2016.5132

15. Liu X, Zhou Q-G, Zhu X-C, Xie L, Cai B-C. Components of fangji huangqi tang on the treatment of nephrotic syndrome by using integrated metabolomics based on"correlations between chemical and metabolic profiles”. Front Pharmacol. 2019;10:1261. doi:10.3389/ fphar.2019.01261

16. Perez G. Anti-inflammatory activity of compounds isolated from plants. Sci World J. 2001;1:713-784. doi:10.1100/tsw.2001.77

17. Choi H-S, Kim H-S, Min KR, et al. Anti-inflammatory effects of fangchinoline and tetrandrine. J Ethnopharmacol. 2000;69:173-179. doi:10.1016/S0378-8741(99)00141-5

18. Chen H, Wang Y, Zhu C, Zhang M. Clinical and pathological observation of the effect of individualization combined by traditional Chinese medicine and western medicine and sequenced therapy on IgA nephrology. J Nephropathy Integr Chin West Med. 2004;5:261-265.

19. Wang Y-J, He L-Q, Sun W, et al. Optimized project of traditional Chinese medicine in treating chronic kidney disease stage 3 : a multicenter double-blinded randomized controlled trial. $J$ Ethnopharmacol. 2012;139:757-764. doi:10.1016/j.jep.2011.12. 009

20. Zhou H-Y, Wang F, Cheng L, Fu L-Y, Zhou J, Yao W-X. Effects of tetrandrine on calcium and potassium currents in isolated rat hepatocytes. World J Gastroenterol. 2003;9:134. doi:10.3748/wjg.v9. i1.134

21. Liu Q-Y, Karpinski E, Pang P. Tetrandrine inhibits both $\mathrm{T}$ and $\mathrm{L}$ calcium channel currents in ventricular cells. J Cardiovasc Pharmacol. 1992;20:513-519. doi:10.1097/00005344-199210000-00 001

22. Liu Q-Y, Karpinski E, Rao M-R, Pang P. Tetrandrine: a novel calcium channel antagonist inhibits type I calcium channels in neuroblastoma cells. Neuropharmacology. 1991;30:1325-1331. doi:10.1016/00283908(91)90030-F

23. Takemura H, Imoto K, Ohshika H, Kwan CY. Tetrandrine as a calcium antagonist. Clin Exp Pharmacol Physiol. 1996;23:751-753. doi:10.11 11/j.1440-1681.1996.tb01772.x

24. Qi L, Xian-Yang Z, Yu-Feng X, Yue D, Zhi-Feng W. Tetrandrine inhibits migration and invasion of rheumatoid arthritis fibroblast-like synoviocytes through down-regulating the expressions of Rac1, Cdc42, and RhoA GTPases and activation of the PI3K/Akt and JNK signaling pathways. Chin J Nat Med. 2015;13:831-841. doi:10.1016/S1875-5364(15)30087-X

25. Zhang Z, Liu T, Yu M, Li K, Li W. The plant alkaloid tetrandrine inhibits metastasis via autophagy-dependent $\mathrm{Wnt} / \beta$-catenin and metastatic tumor antigen 1 signaling in human liver cancer cells. $J$ Exp Clin Cancer Res. 2018;37:7. doi:10.1186/s13046-018-0678-6

26. Huang T, Xu S, Deo R, et al. Targeting the Ca2+/Calmodulin-dependent protein kinase II by Tetrandrine in human liver cancer cells. Biochem Biophys Res Commun. 2019;508:1227-1232. doi:10.1016/j.bbrc.2018. 12.012

27. Qian Y, Huang Y. Effects of tetrandrine on rabbit platelet aggregation, thromboxane A2 generation and calmodulin activity. Zhongguo Yao Li Xue Bao. 1989;10:61-65.

28. Gu C, Zhou G, Noble NA, Border WA, Cheung AK, Huang Y. Targeting reduction of proteinuria in glomerulonephritis: maximizing the antifibrotic effect of valsartan by protecting podocytes. $J$ Renin Angiotensin Aldosterone Syst. 2014;15:177-189. doi:10.1177/14703 20312466127 
29. Huang F, Wang Q, Ma X, Wu L, Guo F, Qin G. Valsartan inhibits amylin-induced podocyte damage. Microvasc Res. 2016;106:10 1-109. doi:10.1016/j.mvr.2016.04.007

30. Klein RR, Bourdon DM, Costales CL, et al. Direct activation of human phospholipase $\mathrm{C}$ by its well known inhibitor u73122. J Biol Chem. 2011;286:12407-12416. doi:10.1074/jbc.M110.191783

31. Tozzi A, Durante V, Bastioli G, et al. Dopamine D2 receptor activation potently inhibits striatal glutamatergic transmission in a G2019S LRRK2 genetic model of Parkinson's disease. Neurobiol Dis. 2018;118:1-8. doi:10.1016/j.nbd.2018.06.008

32. Wang S, Chen C, Su K, et al. Angiotensin II induces reorganization of the actin cytoskeleton and myosin light-chain phosphorylation in podocytes through rho/ROCK-signaling pathway. Ren Fail. 2016;38:268-275. doi:10.3109/0886022X.2015.1117896

33. Zhou G, Cheung AK, Liu X, Huang Y. Valsartan slows the progression of diabetic nephropathy in $\mathrm{db} / \mathrm{db}$ mice via a reduction in podocyte injury, and renal oxidative stress and inflammation. Clin Sci. 2014;126:707-720. doi:10.1042/CS20130223
34. Gao F, Yao M, Cao Y, Liu S, Liu Q, Duan H. Valsartan ameliorates podocyte loss in diabetic mice through the Notch pathway. Int $\mathrm{J} \mathrm{Mol}$ Med. 2016;37:1328-1336. doi:10.3892/ijmm.2016.2525

35. Wang Q, Li R, Li W, Wang L. Protective effect of valsartan on podocyte injury in rats with diabetic nephropathy. Am J Life Sci. 2018;6:47-51. doi:10.11648/j.ajls.20180603.12

36. Zhang J, Hu X, Wang S, Zhang Y, Yang H. Protective effects of low-dose rapamycin combined with valsartan on podocytes of diabetic rats. Int J Clin Exp Med. 2015;8:13275.

37. Schlondorff J, Del Camino D, Carrasquillo $R$, et al. TRPC6 $\mathrm{m}$ utations associated with focal segmental glomerulosclerosis cause constitutive activation of NFAT-dependent transcription. Am J Physiol Cell P Hysiol. 2009;296(3):C558-C569. doi:10.1152/ ajpcell.00077.2008

38. Clark K, Middelbeek J, van Leeuwen FN. Interplay between TRP channels and the cytoskeleton in health and disease. Eur J Cell Biol. 2008;87(8-9):631-640. doi:10.1016/j.ejcb.2008.01.009

\section{Publish your work in this journal}

Drug Design, Development and Therapy is an international, peerreviewed open-access journal that spans the spectrum of drug design and development through to clinical applications. Clinical outcomes, patient safety, and programs for the development and effective, safe, and sustained use of medicines are a feature of the journal, which has also been accepted for indexing on PubMed Central. The manuscript management system is completely online and includes a very quick and fair peer-review system, which is all easy to use. Visit http://www. dovepress.com/testimonials.php to read real quotes from published authors. 